\title{
Bilateral basal ganglia haemorrhage following motor vehicle accident: $A$ Case report
}

\author{
Pankaj Gupta ${ }^{1}$, R.S. Mittal ${ }^{2}$, Devendra Purohit ${ }^{3}$, Jitendra Singh \\ Shekhawat ${ }^{4}$, Ugan Singh Meena ${ }^{5}$
}

${ }^{1}$ Senior Resident, Dept. Of Neurosurgery, S.M.S. Medical College, Jaipur, Rajasthan ${ }^{2}$ Dept. Of Neurosurgery, S.M.S. Medical College, Jaipur, Rajasthan ${ }^{3}$ Professor, Dept. Of Neurosurgery, S.M.S. Medical College, Jaipur, Rajasthan ${ }^{4}$ Assist. Professor, Dept. Of Neurosurgery, S.M.S. Medical College, Jaipur, Rajasthan ${ }^{5}$ Senior Resident, Dept. Of Neurosurgery, S.M.S. Medical College, Jaipur, Rajasthan

\begin{abstract}
Bilateral basal ganglia haemorrhage following motor vehicle accident is extremely rare in field of neurosurgery. This is presumed to be secondary to rupture of lenticulostriate or Anterior Choroidal artery by shearing, as a result of acceleration/deceleration forces. Although the exact mechanism is still not known. Isolated bilateral basal ganglia haemorrhage carries favourable prognosis. We report a rare case of 50 years old lady having bilateral basal ganglia haemorrhage with diffuse axonal injury following motor vehicle accident. Patient was managed conservatively and improved.
\end{abstract}

Key words: Motor Vehicle Accident, Basal Ganglia, Diffuse Axonal Injury.

\section{Introduction}

Post-traumatic bilateral basal ganglia haemorrhage is extremely rare entity. It consists of haemorrhagic lesions in basal ganglia (Caudate nucleus, Putamen, Globus
Pallidus), Internal Capsule or Thalamus. (3, 5, 6) Incidence of post-traumatic basal ganglia bleed is $3 \%$ after motor vehicle accident (MVA) in clinical series, while it is $10-12 \%$ in autopsy series. $(3,6)$ Traumatic basal ganglia haemorrhage (TBGH) is classified as 'Large' if it is $>2 \mathrm{~cm}$ in diameter, and as 'Small' if $<2 \mathrm{~cm}$ in diameter. The exact mechanism is still not clear but, it is believed to arise from a shear strain in the ganglionic region. This shear strain is produced by acceleration/ deceleration forces during high velocity injury, which produces rupture of lenticulostriate or Anterior Choroidal artery, hence bilateral basal ganglia bleed. (1) There is single case report of bilateral basal ganglia haemorrhage following lightning strike, where it is related to preferential conduction pathways through the brain. Bilateral TBGH can occur as isolated lesion or in association with Diffuse Axonal Injury (DAI), cerebral contusions, Subdural Hematoma (SDH), Extradural Hematoma (EDH) or, Subarachnoid Haemorrhge (SAH). 
(7) Bilateral TBGH carries dismal Prognosis. Isolated TBGH has good outcome.

\section{Case Report}

A 50 years old female patient was admitted to our institute with history of MVA. At the time of admission, she was unconscious (GCS $=7$ ) and pupils were $4 \mathrm{~mm}$ size, reacting normally to light. Non-contrast CT head showed bilateral (mirror-image) basal ganglia haemorrhage, tentorial bleed, posterior interhemispheric bleed and scalp hematoma over left frontal region. There was no evidence of ventricular compression, effacement of basal cisterns, mass effect or midline shift. Cerebral CT angiography was within normal limits and routine blood investigations did not reveal any evidence of coagulation disorder.

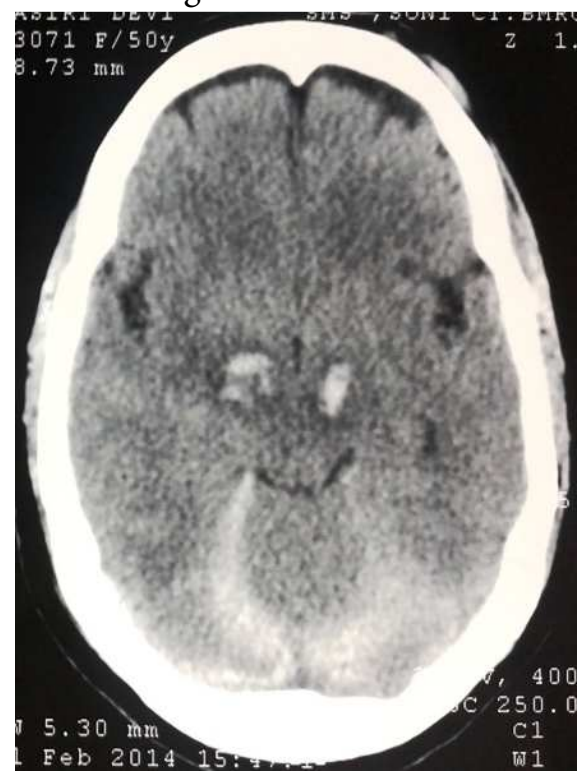

Figure 1 - NCCT Head showing mirror image acute haemorrhage in bilateral basal ganglia region with tentorial bleed and extra-calvarial scalp hematoma
Patient was intubated immediately to avoid aspiration of secretions as GCS score was 7 . Later on tracheostomy was done after 24 hours. Patient was kept on Mannitol, antibiotics and anticonvulsants. On 8th day, patient's GCS was 10 with left sided hemiparesis. She was discharged on 14th day after decannulation of tracheostomy tube, with residual left hemiparesis.

\section{Discussion}

Bilateral TBGH is extremely rare but a serious complication of head injury. $(1,2)$ TBGH is usually small and located in the region of lentiform nucleus or external capsule, while spontaneous hypertensive basal ganglia bleed is principally located in the region of Thalamus and Internal capsule. To the best of our knowledge, only 2 cases have been reported till date.

Regarding mechanism of TBGH, Mosberg \& Lindenberg demonstrated traumatic tear of a pallidal branch of anterior choroidal artery as the source of origin of pallidal hematoma in an autopsy case. (1) When there is a strong impact to the vertex, forehead or occiput and directed towards the tentorium, there would be a shift of brain, through the tentorial notch with stretching and tearing of vessels by shearing forces, resulting in basal ganglia haemorrhage.

The final prognosis of patients with TBGH is strongly related to existence of associated DAI. In our case, DAI was considered as patient was unconscious after injury and CT findings were also consistent with DAI. 
DOI: 10.2478/romneu-2014-0028

In addition, differentiation of traumatic from spontaneous basal ganglia bleed is very important from medicolegal point of view, since the basal ganglia is a frequent site of spontaneous hypertensive bleed. TBGH are dynamic lesions and tend to expand in volume during acute post-traumatic period, hence there is importance of serial CT scanning.

Surgical options available for these cases are open surgery or, USG/CT guided stereotactic aspiration but, the outcome of most surgically treated patients was poor. (4, 8) The absolute indication of surgery in these cases are, volume of lesion $>25 \mathrm{ml}$ and increasing size of hematoma with deterioration of neurological condition of patient. In aaddition, medical and supportive treatment includes ICP monitoring, elective ventilation and close monitoring in ICU. (6)

The good prognostic factors in TBGH are younger age $(<60 \mathrm{yrs})$, small lesions $(<2 \mathrm{~cm})$, GCS $>9$ at the time of admission, isolated lesion. The bad prognostic factors are old age (>60yrs), large lesion ( $>2 \mathrm{~cm}$ ), GCS $<7$ at the time of admission, Basal ganglia haemorrhage alongwith associated DAI, EDH, SDH, SAH or cerebral contusions and coexisting medical Comorbidities like Hypertension, Diabetes or coagulation disorders. (7) TBGH carries the tendency of delayed (within 48 hours) expansion of hematoma, which is again a bad prognostic indicator. $(2,7)$

\section{Conclusion}

The TBGH are usually haemorrhagic contusions, as a result of shear forces after MVA. The overall mortality is high and morbidity is considerable in survivors. Patients with isolated TBGH do well with conservative management.

\author{
Footnotes \\ Source of support: Nil. \\ Conflicts of interest: None declared.

\section{Correspondence:} \\ Dr. Devendra Purohit (Mch.) \\ Professor (Neurosurgery) \\ Deptt. Of Neurosurgery, S.M.S. Medical College, \\ Jaipur, Rajasthan, 302004. \\ email:drpankaj.gupta@yahoo.co.in \\ devendrapurohit@rediffmail.com
}

\section{References}

Mosberg WH Jr, Lindenberg R. Traumatic hemorrhage from the anterior choroidal artery.J Neurosurg 1959; 16: 209-21.

Yanaka K, Egashira T, Maki Y, et al. Bilateral traumatic hemorrhage in the basal ganglia: report of two cases.No Shinkei Geka 1991;19: 369-73.

Boto GR, Lobeto RD, Rivas J. Basal ganglion hematoma in severely head injured patients: clinicoradiological analysis of 37cases.J Neurosurg 2001; 94:224-32.

Kim SH, Song SH, Youm JY, Kim Y: Clinical analysis of the traumatic basal ganglia hemorrhage. J Korean Neurosurg Soc 25: 1223-1229, 1996.

Crooks DA. Pathogenesis ana biomechenics of traumatic intracranial hemorrhages. Virchows Arch A Pathol Anat Histopathol 1991;418:479-483.

Yamakawa N, Furuno M, Okada M et al. traumatic basal ganglia haemorrhage: report of 7 cases. J Clin Neurosci 1995; 2:55-58.

Kimura M, Sobata E, Suzuki S. Traumatic basal ganglion (caudate) with favourable prognosis: report of two cases. No Shinkei Geka 1994;22:155-158.

8. Yanagawa $\mathrm{Y}$, Kiyozumi T, Terai $\mathrm{C}$ et al. A case of traumatic hematoma in the basal ganglia: successful drainage with ultra-sound guided aspiration surgery via burr hole. No Shinkei Geka 1997;25:1105-1108 (Jpn). 